[0212-7199 (2006) 23: 12; pp 596-601] ANALES DE MEDICINA INTERNA Copyright (C) 2006 ARAN EDICIONES, S.L.

AN. MED. INTERNA (Madrid) Vol. 23, N. ${ }^{\circ} 12$, pp. 596-601, 2006

\section{Influencia de los diferentes regímenes de administración de los bifosfonatos sobre la tolerabilidad}

\author{
M. SOSA HENRÍQUEZ, M. J. GÓMEZ DE TEJADA ROMERO' \\ Universidad de Las Palmas de Gran Canaria. Facultad de Ciencias de la Salud. Grupo \\ de Trabajo en Osteoporosis y Metabolismo Mineral. Hospital Universitario Insular. \\ Las Palmas de Gran Canaria. ${ }^{I}$ Departamento de Medicina. Universidad de Sevilla
}

INFLUENCE OF THE DIFFERENT BISPHOSPHONATES DOSE REGIMENS ON ITS TOLERABILITY

\begin{abstract}
RESUMEN
La osteoporosis es la enfermedad ósea más frecuente cuya complicación clínica son las fracturas, siendo la vertebral la más prevalerte. Disponemos hoy en día de un número de fármacos que reducen la aparición de nuevas fracturas y entre estos ocupan un papel fundamental los bifosfonatos.

Sin embargo para que se produzca la reducción de fracturas, el tratamiento debe realizarse correctamente a largo plazo, y una gran proporción de pacientes abandonan la medicación al cabo de pocos meses por distintas razones.

La introducción en el arsenal terapéutico contra la osteoporosis de bifosfonatos que permiten la administración más separada en el tiempo, semanal e incluso mensualmente, ha mejorado notablemente la adherencia al tratamiento.
\end{abstract}

PALABRAS CLAVE: Osteoporosis. Fracturas. Bifosfonatos. Adherencia semanal y mensual.

\begin{abstract}
Osteoporosis is the most prevalent metabolic bone disease and fractures are its clinical complication. We have nowadays some drugs that reduce the incidence of new fractures: Bisphosphonates.

Nevertheless, treatment must be taken properly in the long run to reduce the incidente of new fractures and a few months alter starting the treatment, a great number of patients stop it because of different reasons.

The introduction of new bisphosphonates that can be taken weekly or even better monthly, has improved notably the adherence and compliance to osteoporosis treatment.
\end{abstract}

KEY WORDS: Osteoporosis. Fractures. Bisphosphonates. Adherence weekly and monthly.

Sosa Henríquez, M, Gómez de Tejada Romero MJ. Influencia de los diferentes regímenes de administración de los bifosfonatos sobre la tolerabilidad. An Med Interna (Madrid) 2006; 23: 596-601.

\section{INTRODUCCIÓN. LA IMPORTANCIA DE LA OSTEOPOROSIS}

La osteoporosis es una enfermedad que afecta a todo el esqueleto y que produce fracturas como principal complicación clínica. Existe una alteración de la cantidad del hueso, que se manifiesta como una pérdida de la densidad mineral ósea, y una alteración de la calidad ósea, producida por una alteración de la microarquitectura (1).

Las fracturas osteoporóticas pueden producirse en cualquier hueso del esqueleto, aunque los más comúnmente afectados son las vértebras, el radio distal (fractura de Colles) y la extremidad proximal del fémur (fractura de cadera) $(2,3)$. Los pacientes con fracturas osteoporóticas pueden tienen manifestaciones clínicas como dolor, pérdida de estatura, cifosis y/o escoliosis (4), así como una menor calidad de vida $(5,6)$ y mayor mortalidad (7) que el resto de la población.
Las consecuencias de la osteoporosis son muy amplias, y se extienden a las esferas física, psicosocial y económica del paciente, produciendo un notable impacto también en su familia y en la comunidad. Una fractura osteoporótica es prácticamente siempre el resultado final de un traumatismo que ocurre en un hueso que tiene previamente afectada su resistencia. El trauma capaz de desencadenar una fractura puede tener una magnitud muy variada, desde una caída producida de una altura elevada hasta simplemente flexionar la espalda para elevar una carga.

La incidencia de fracturas es alta en los pacientes que tienen osteoporosis y aumenta con la edad. La probabilidad de que una persona de 50 años desarrolle una fractura de cadera durante el resto de su vida es del $14 \%$ en una mujer de raza blanca y del 6\% en un varón de la misma raza (8). Las personas de raza negra tienen un menor riesgo de padecer fracturas

Trabajo aceptado: 24 de julio de 2006 
osteoporóticas y, así, en la población afro-americana estadounidense, el riesgo antes mencionado es del $6 \%$ en las mujeres y del $3 \%$ en los varones. En un estudio efectuado en nuestro país, en Gran Canaria, durante 5 años, se observó que el 90\% de los pacientes que sufrían una fractura de cadera provenían de sus domicilios, pero que, en el momento de su alta, menos de la mitad volvían a ellos, siendo la mayoría remitidos a centros de rehabilitación y de crónicos (9). Además de la elevada morbilidad, las fracturas osteoporóticas tienen una notable mortalidad, tanto las fracturas vertebrales (10) como las de cadera. Al año de haberse producido la fractura de cadera, han fallecido el $30 \%$ de los pacientes y a los dos años cerca del $40 \%$ (11). La fractura vertebral y de cadera constituyen un problema para la mujer sobre todo entre los 70 y 80 años, mientras que la fractura de la extremidad distal del radio (fractura de Colles) se produce entre los 50 y 70 años.

\section{¿QUÉ OCURRE CUANDO SE SUSPENDE EL TRATAMIENTO?}

El arsenal terapéutico de que disponemos en la actualidad para el tratamiento de la osteoporosis ha cambiado espectacularmente si lo comparamos con el existente tan sólo 10 años atrás. Además, la aparición de nuevos estudios y meta-análisis ha permitido también establecer la verdadera utilidad de fármacos disponibles en el pasado y que hoy en día parecen ser más perjudiciales que beneficiosos, como ocurre con el fluoruro sódico o el tratamiento hormonal sustitutivo.

En estudios efectuados bajo los exigentes criterios de la Medicina Basada en la Evidencia, varios tratamientos han demostrado ser eficaces en la reducción del riesgo de nuevas fracturas (Tabla I). El seguimiento de las pacientes ha sido habitualmente de 3 años, aunque en algunos casos se han realizado seguimientos a más largo plazo. Sin embargo, una vez concluido el estudio y aplicando sus resultados a la práctica clínica, desconocemos cuánto tiempo debería mantenerse el tratamiento farmacológico de la osteoporosis, y si deberían o no efectuarse períodos de descanso.

\section{TABLA I}

FÁRMACOS CON EFICACIA COMPROBADA EN LA REDUCCIÓN DE FRACTURAS OSTEOPORÓTICAS

\begin{tabular}{llll}
\hline Fármaco & Año & Nombre estudio & Cita \\
\hline Etidronato & 1990 & & 12 \\
Alendronato & 1995,1996 & FIT & 13,14 \\
Risedronato & $1999,2000,2001,2003$ & VERT, HIP & $15-18$ \\
Raloxifeno & 1999 & MORE & 19 \\
Calcitonina & 2000 & PROOF & 20 \\
Estroncio & 2002,2005 & TROPOS, ESTRATOS & 21,22 \\
PTH & 2001 & FTI & 23 \\
Estrógenos & 2002,2004 & WHI & 24,25 \\
Ibandronato & $2003,2005,2006$ & MOBILE & $26-28$ \\
\hline
\end{tabular}

La osteoporosis es una enfermedad crónica y la pérdida de masa ósea se produce inexorablemente después de suspenderse el tratamiento. Esto ha sido comprobado en las cohortes de "seguimiento" de estos mismos estudios. Así, por ejemplo, la prolongación del estudio FIT con alendronato a 10 años (29) mostró que las mujeres que habían suspendido el tratamiento tenían una mayor incidencia tanto de fracturas vertebrales $(4,6 \%$ frente a $2,2 \%, \mathrm{p}<0,001)$ como de no vertebrales $(13,0 \%$ frente a $10,3 \%, \mathrm{p}=0,013)$, así como una mayor pérdida de masa ósea. En otro estudio de seguimiento a largo plazo en el que se combinaba alendronato y terapia hormonal sustitutiva se observó que, al suspender los estrógenos, las pacientes perdían una media del 4,5\% de densidad mineral ósea en la columna y del $1,8 \%$ en la cadera, junto con un incremento de los marcadores bioquímicos de remodelado óseo, hasta alcanzar los valores previos al inicio del tratamiento (30). En el caso particular de la terapia hormonal sustitutiva, otros estudios han confirmado que la suspensión del tratamiento produce una pérdida de la densidad mineral ósea hasta el punto de que, en algunos estudios epidemiológicos, se ha comprobado que el riesgo de fractura que adquieren estas pacientes tras suspender el tratamiento es el mismo que tienen aquellas mujeres que nunca comenzaron la terapia estrogénica (31-34). Prácticamente lo mismo se ha observado al suspender el raloxifeno. En un estudio efectuado por Neele y cols. (35) se constató que aquellas mujeres postmenopáusicas que habían suspendido el raloxifeno, al año tenían una pérdida del 2,4\% en la columna lumbar y del 3,0\% en el cuello femoral. Con el fluoruro sódico también se comprobó que en el $73 \%$ de los pacientes que habían suspendido el fármaco la densidad mineral ósea disminuía hasta llegar a los valores que tenían antes de comenzar el tratamiento (36).

Algunos estudios han observado un cierto efecto "residual" de la medicación después de suspender el fármaco. Así, por ejemplo, con PTH se ha descrito una reducción del riesgo de fractura vertebral, tanto en hombres como en mujeres, después de suspenderse el tratamiento con el fármaco, al prolongarse el seguimiento hasta 40 meses $(37,38)$. Con el alendronato se ha visto que al suspender el tratamiento existía también este efecto residual, apreciándose una gradual pérdida del mismo, determinada tanto por densidad mineral ósea como por los marcadores bioquímicos de remodelado óseo (39). Este efecto también se ha observado con el risedronato, pues al cabo de 6 meses tras la suspensión de este fármaco se observó un regreso a los valores previos al tratamiento de los marcadores bioquímicos de remodelado óseo (40).

Pero el problema más importante radica en que la toma incorrecta del tratamiento para la osteoporosis implica un aumento del riesgo de nuevas fracturas por fragilidad. Se han publicado hasta el momento 2 estudios corroborando este hecho. Así, McCombs y cols. (41), analizando todas la terapias para la osteoporosis (tratamiento hormonal sustitutivo, bifosfonatos y raloxifeno) indicadas a 58.109 pacientes y seguidas durante 245 días, observaron que para obtener una reducción del riesgo de fractura era necesario que el tratamiento se hubiera tomado correctamente al menos durante 1 año. En el otro estudio, realizado en Canadá en 11.249 mujeres que tomaban etidronato, alendronato o risedronato, se observó que aquéllas que tomaban el fármaco correctamente tenían un $16 \%$ menos de fracturas que las que no lo hacían (42).

También debe tenerse en cuenta que prácticamente la totalidad de los tratamientos disponibles en la actualidad previenen la aparición de nuevas fracturas entre un 40-60\% (43). Lo cual quiere decir que, aun actuando el fármaco de forma adecuada, aproximadamente el $50 \%$ de las pacientes pueden tener una nueva fractura, incluso tomando correctamente la medicación. 


\section{EL PROBLEMA DE LA ADHERENCIA AL TRATAMIENTO EN LA OSTEOPOROSIS}

Por todo lo antes mencionado, que los pacientes afectos de osteoporosis cumplan las instrucciones de administración del tratamiento, a la dosis e intervalos adecuados, es una necesidad perentoria. También se ha establecido que para que la medicación sea eficaz para reducir el riesgo de nuevas fracturas, en la mayor parte de los casos debe tomarse como mínimo durante 1 año $(44,45)$, aunque con el risedronato se ha observado reducción de este riesgo a los 6 meses (17). Sin embargo, en la práctica clínica diaria, la realidad no es así. Se ha estimado que aproximadamente el $20 \%$ de los pacientes a los que se les indica un tratamiento para la osteoporosis no llega ni siquiera a comenzarlo (46), y entre los que lo inician, las cifras de abandono son muy variables en las distintas series publicadas, dependiendo del fármaco, el período de tiempo analizado, el nivel socioeconómico e incluso el país (47). Las causas que pueden justificar un mal cumplimiento o abandono del tratamiento son muy variadas (Tabla III).

Por citar sólo algunos ejemplos, en un estudio italiano (48) realizado con casi 10.000 mujeres postmenopáusicas y con un seguimiento de 14 meses, el 19,1\% había suspendido el tratamiento antes de la siguiente revisión densitométrica, y de ellas más de la mitad lo había abandonado en los primeros 6 meses. El régimen terapéutico más estudiado con respecto a su cumplimiento es el tratamiento hormonal sustitutivo, y las cifras de adherencia al mismo oscilan entre el 8 y el 70\% (49-53), disminuyendo con el tiempo (54). Pero además, recientemente, tras la publicación de los resultados del WHI $(24,25)$, los abandonos de la terapia hormonal sustitutiva han aumentado drásticamente. En un estudio realizado en nuestro medio obtuvimos que, entre las mujeres que recibían terapia estrogénica, al cabo de 3 años habían abandonado el tratamiento más de la mitad de las pacientes, independientemente de que tuvieran o no osteoporosis (55). Lo mismo se ha observado con los bifosfonatos. Un trabajo efectuado por Lombas y cols. (56) con alendronato, administrado diariamente a 401 mujeres posmenopáusicas con valores densitométricos de osteopenia u osteoporosis, mostró que el $13 \%$ no llegó a comenzar el tratamiento, que el 51\% lo había suspendido al cabo de un año y hasta el $70 \%$ al cabo de 2 años.

\begin{tabular}{|c|c|c|}
\hline \multicolumn{3}{|c|}{ TABLA II } \\
\hline \multicolumn{3}{|c|}{$\begin{array}{c}\text { GENERACIONES Y POTENCIA RELATIVA DE LOS } \\
\text { BIFOSFONATOS }\end{array}$} \\
\hline Estructura química & Nombre genérico & Potencia antirresortiva \\
\hline \multicolumn{3}{|l|}{ Primera generación } \\
\hline $\begin{array}{l}\text { Cadena corta } \\
\text { alquil/aldehido }\end{array}$ & $\begin{array}{l}\text { Etidronato } \\
\text { Clodronato }\end{array}$ & $\begin{array}{l}1 \\
10\end{array}$ \\
\hline \multicolumn{3}{|l|}{ Segunda generación } \\
\hline Grupo amino-terminal & $\begin{array}{l}\text { Tiludronato } \\
\text { Pamidronato } \\
\text { Alendronato }\end{array}$ & $\begin{array}{l}10 \\
100 \\
100-1.000\end{array}$ \\
\hline Tercera generación & & \\
\hline Cadena lateral cíclica & $\begin{array}{l}\text { Risedronato } \\
\text { Ibandronato } \\
\text { Zoledronato }\end{array}$ & $\begin{array}{l}1.000-10.000 \\
1.000-10.000 \\
>10.000\end{array}$ \\
\hline
\end{tabular}

La OMS ha descrito la pobre adherencia a la medicación como un problema mundial de una gran magnitud (57) también en el campo de la osteoporosis (58). Precisamente, la osteoporosis puede considerarse como una enfermedad "silente", sin síntomas, y por lo tanto el paciente no percibe subjetivamente los beneficios del tratamiento. Las determinaciones de densidad mineral ósea o de los marcadores bioquímicos de remodelado óseo, únicos parámetros medibles en la clínica diaria para observar el seguimiento de la enfermedad, no son fácilmente accesibles para toda la población, por lo que los pacientes no tienen medio de objetivar, de alguna manera, su evolución. El tratamiento debe mantenerse durante muchos años con la finalidad de evitar la aparición de fracturas. Por ello, muchos pacientes terminan suspendiendo la medicación.

\begin{tabular}{c} 
TABLA III \\
POSIBLES RAZONES POR LAS QUE LAS PACIENTES NO \\
TOMAN ADECUADAMENTE LA MEDICACIÓN PARA LA \\
OSTEOPOROSIS \\
\hline
\end{tabular}

Relacionadas con el paciente

Pérdida de memoria relacionada con la edad

Demencia

Ausencia de concienciación de la necesidad de una toma constante

Escasos recursos económicos. Nivel cultural

Polimedicación

Miedo a la medicación

Escasa información

Situación clínica (fracturas, enfermedad de base concomitante, esteroides)

Relacionadas con la enfermedad

Ausencia de síntomas específicos

Ausencia de un buen marcador que monitorice la respuesta al tratamiento

\section{Relacionadas con el fármaco \\ Efectos secundarios \\ Precio}

Comodidad en su administración

Intervalos entre dosis

Efectos "beneficiosos" colaterales: analgesia, reducción riesgo de cáncer de mama

Prospecto

\section{Relacionadas con el sistema sanitario}

Grado de colaboración/implicación del médico de atención primaria

Presión de las administraciones sanitarias para el control del gasto sanitario

INFLUENCIA DE LOS DIFERENTES REGÍMENES DE ADMINISTRACIÓN DE LOS BIFOSFONATOS SOBRE LA TOLERABILIDAD

Los bifosfonatos constituyen un grupo de fármacos ampliamente utilizado en el campo de las enfermedades metabólicas óseas en general y de la osteoporosis en particular. 
Muchos estudios han confirmado su utilidad en la reducción del riesgo de fracturas osteoporóticas (12-18,26-29) y en la actualidad se consideran fármacos de primera elección para el tratamiento de la osteoporosis $(59,60)$.

Los bifosfonatos son muy diferentes entre sí, tanto en sus vías de administración como en sus propiedades farmacocinéticas y potencias (61-65) (Tabla II). Posiblemente por ello, los resultados clínicos son diferentes, como también lo son sus efectos secundarios. Todos tienen en común una muy escasa absorción en el tracto gastrointestinal, hasta el extremo de que en condiciones óptimas se absorbe menos del $1 \%(63,66)$, que se convierte en nula cuando se administra concomitantemente algún tipo de alimento u otro fármaco.

Los aminobifosfonatos con los que existe una mayor experiencia en el tratamiento de la osteoporosis son el alendronato y el risedronato. Ambos han demostrado la reducción del riesgo de fracturas osteoporóticas, tanto vertebrales, como de cadera y no vertebrales en general $(12-18,61)$. Comenzaron a administrarse por vía oral, en ayunas y ortostatismo para favorecer la absorción gastrointestinal y evitar la aparición de efectos secundarios, especialmente la esofagitis $(63,66)$. La administración diaria y los efectos secundarios hicieron que la tasa de abandonos fuera relativamente elevada. Por ello se desarrollaron dosificaciones semanales, con el objetivo de disminuir los efectos secundarios y de esta manera aumentar la adherencia al tratamiento. Varios estudios confirmaron la bioequivalencia entre la dosis diaria y la semanal (67-69). Al efectuarse posteriormente estudios sobre la adherencia al tratamiento comparando ambos períodos de administración, se observó que la ingesta semanal producía una disminución del número de abandonos, el cual aún seguía siendo elevado. Así, en un trabajo efectuado en Alemania por Bartl y cols. (70) en 288 mujeres que iniciaron tratamiento con alendronato, la mitad diariamente y la otra mitad semanalmente, se observó que a los 6 meses habían abandonado el tratamiento el 59\% del grupo diario y el $43,7 \%$ del grupo semanal. Cuando el seguimiento se prolongó hasta 1 año, continuaban tomando correctamente el fármaco el $27,8 \%$ del grupo diario y el $46,5 \%$ del semanal. En otro estudio efectuado en EE.UU., Cramer y cols. (71) observaron que al cabo de un año, la persistencia en el tratamiento con bifosfonatos fue del $31,7 \%$ en aquellas mujeres que lo tomaban diariamente y del $44,2 \%$ en aquéllas que lo tomaban semanalmente.

Varios estudios indican que los pacientes afectos de osteoporosis prefieren la toma semanal a la diaria $(72,73)$. No cabe duda de que la administración semanal de los bifosfonatos ha aumentado la adherencia al tratamiento de la osteoporosis, pero en líneas generales, la toma correcta de la medicación sigue siendo aún baja. Por ello la reciente aparición de un fármaco con una única toma mensual, el ibandronato, puede contribuir a aumentar la adherencia al tratamiento de la osteoporosis.

\section{CONCLUSIONES}

La falta de adherencia al tratamiento en las enfermedades crónicas, como es el caso de la osteoporosis, constituye un problema sanitario de primera magnitud, tal y como ha expuesto la OMS. Los bifosfonatos son un grupo de fármacos de primera elección en el tratamiento de la osteoporosis ya que reducen la aparición de nuevas fracturas por fragilidad a largo plazo. La administración semanal, en lugar de diaria, de alendronato y risedronato supuso una notable mejoría en la adherencia al tratamiento. Cabe esperar que el desarrollo de formulaciones que se administren de una manera más espaciada, por ejemplo mensualmente como ocurre con el ibandronato, contribuyan a aumentar el cumplimiento y la adherencia.

\section{Bibliografía}

1. National Institutes of Health (USA). Consensus Development Panel on Osteoporosis Prevention, Diagnosis, and Therapy. NIH Consensus Development Panel on Osteoporosis. JAMA 2001; 285: 785-95.

2. Walker-Bone K, Walter G, Cooper C. Recent developments in the epidemiology of osteoporosis. Curr Opin Rheumatol 2002; 14: 411-5.

3. Cummings SR, Melton LJ. Epidemiology and outcomes of osteoporotic fractures. Lancet 2002; 359: 1761-7.

4. Truumees E. Medical consequences of osteoporotic vertebral compression fractures. Instr Course Lect 2003; 52: 551-8.

5. Lips P, Cooper C, Agnusdei D, Caulin F, Egger P, Johnell O, et al. Quality of life in patients with vertebral fractures: validation of the Quality of Life Questionnaire of the European Foundation for Osteoporosis (QUALEFFO). Working Party for Quality of Life of the European Foundation for Osteoporosis. Osteoporos Int 1999; 10: 150-60.

6. Oleksik A, Lips P, Dawson A, Minshall ME, Shen W, Cooper C, et al. Health-related quality of life in postmenopausal women with low BMD with or without prevalent vertebral fractures. J Bone Miner Res 2000; 15: $1384-92$

7. Davidson CW, Merrilees MJ, Wilkinson TJ, McKie JS, Gilchrist NL. Hip fracture mortality and morbidity. Can we do better? N Z Med J 2001; 114: 329-32.

8. Melton LJ III. Epidemiology of osteoporosis. Baillieres Clin Obstet Gynaecol 1991; 5: 785-805.

9. Sosa M, Navarro R, Arbelo A. Fractura de cadera: la realidad española. En: Díaz Curiel M, ed. Actualización de osteoporosis. Madrid: FHOE-
MO, 2001; 13-22.

10. Melton LJ III. Excess mortality following vertebral fracture. J Am Geriatr Soc 2000; 48: 338-9.

11. Sosa Henríquez M. La fractura osteoporótica de cadera en España. Rev Esp Enf Metab Óseas 1993; 2: 41-4.

12. Storm T, Thamsborg G, Steiniche T, Genant HK, Sorensen OH. Effect of intermittent cyclical etidronate therapy on bone mass and fracture rate in women with postmenopausal osteoporosis. N Engl J Med 1990; 322: $1265-71$.

13. Liberman UA, Weiss SR, Broll J, Minne HW, Quan H, Bell NH, et al. Effect of oral alendronate on bone mineral density and the incidence of fractures in postmenopausal osteoporosis. The Alendronate Phase III Osteoporosis Treatment Study Group. N Engl J Med 1995; 333: 1437 43.

14. Black DM, Cummings SR, Karpf DB, Cauley JA, Thompson DE, Nevitt MC, et al. Randomised trial of effect of alendronate on risk of fracture in women with existing vertebral fractures. Fracture Intervention Trial Research Group. Lancet 1996; 348: 1535-41.

15. Watts NB, Josse RG, Hamdy RC, Hughes RA, Manhart MD, Barton I, et al. Risedronate Prevents New Vertebral Fractures in Postmenopausal Women at High Risk. J Clin Endocrinol Metab 2003; 88: 542-9.

16. Reginster JY, Minne HW, Sorenson OH, Hooper M, Roux C, Brandi ML, et al. Randomized trial of the effects of risedronate on vertebral fractures in women with established postmenopausal osteoporosis. Osteoporos Int 2000; 11: 83-91. 
17. Harris ST, Watts NB, Genant HK, McKeever CD, Hangartner T, Keller $\mathrm{M}$, et al. Effects of risedronate treatment on vertebral and nonvertebral fractures in women with postmenopausal osteoporosis. JAMA 1999; 282: $1344-52$.

18. McClung MR, Geusens P, Miller PD, Zippel H, Bensen WG, Roux C, et al. Effect of risedronate on the risk of hip fracture in elderly women. Hip Intervention Program Study Group. N Engl J Med 2001; 344: 333 40.

19. Ettinger B, Black DM, Mitlak BH, Knickerbocker RK, Nickelsen T, Genant HK, et al. Reduction of vertebral fracture risk in postmenopausal women with osteoporosis treated with raloxifene. Results from a 3 year randomized clinical trial. JAMA 1999; 282: 637-45.

20. Chesnut CH III, Silverman S, Andriano K, Genant H, Gimona A, Harris S, et al. A randomized trial of nasal spray salmon calcitonin in postmenopausal women with established osteoporosis: the prevent recurrence of osteoporotic fractures study. PROOF Study Group. Am J Med 2000; 109: 267-76.

21. Reginster JY, Seeman E, De Vernejoul MC, Adami S, Compston J, Phenekos C, et al. Strontium ranelate reduces the risk of nonvertebral fractures in postmenopausal women with osteoporosis: Treatment of Peripheral Osteoporosis (TROPOS) study. J Clin Endocrinol Metab 2005; 90: 2816-22.

22. Meunier PJ, Slosman DO, Delmas PD, Sebert JL, Brandi ML, Albanese C, et al. Strontium Ranelate: Dose-dependent effects in established postmenopausal vertebral osteoporosis - A 2 year randomised placebo controlled trial. J Clin Endocrinol Metab 2002; 87: 2060-66.

23. Neer RM, Arnaud CD, Zanchetta JR, Prince R, Gaich GA, Reginster JY, et al. Effect of parathyroid hormone (1-34) on fractures and bone mineral density in postmenopausal women with osteoporosis. N Engl J Med 2001; 344: 1434-41.

24. Writing Group for the Women Health Initiative Investigators. Risk and benefits of estrogen plus progestin in healthy postmenopausal women. JAMA 2002; 288: 321-33.

25. Anderson GL, Limacher M, Assaf AR, Bassford T, Beresford SA, Black $\mathrm{H}$ et al. Effects of conjugated equine estrogen in postmenopausal women with hysterectomy: the Women's Health Initiative randomized controlled trial. JAMA 2004; 291: 1701-12.

26. Millar PD, McClung MR, Macovei L, Stakkestad JA, Luckey M, Bonvoisin B, et al. Monthly oral ibandronate therapy in postmenopausal osteoporosis: 1-year results from the MOBILE Study. J Bone Miner Res 2005; 20: 1315-22.

27. Reginster JY, Felsenberg D, Cooper C, Stakkestad JA, Miller PD, Kendler DL et al. A new concept for bisphosphonate therapy: a rationale for the development of monthly oral dosing of ibandronate. Osteoporos Int 2006; 17: 159-66.

28. Cooper C, Emkey RD, McDonald RH, Hawker G, Bianchi G, Wilson $\mathrm{K}$, et al. Efficacy and safety of oral weekly ibandronate in the treatment of postmenopausal osteoporosis. J Clin Endocrinol Metab 2003; 88: 4609-15.

29. Ensrud KE, Barrett-Connor EL, Schwartz A, Santora AC, Bauer DC, Suryawanshi $\mathrm{S}$ et al. Randomized trial of effect of alendronate continuation versus discontinuation in women with low BMD: Results from the fracture intervention trial long-term extension. J Bone Miner Res 2004; 19: 1259-69.

30. Greenspan SL, Emkey RD, Bone HG III, Weiss SR, Bell NH, Downs RW Jr, et al. Significant differential effects of alendronate, estrogen, or combination therapy on the rate of bone loss after discontinuation of treatment of postmenopausal osteoporosis. Ann Intern Med 2002; 137: 875-83.

31. McClung M, Wasnich R, Omizo M, Christiansen C, Ravn P, Hosking $\mathrm{D}$, et al. Resolution of effect following alendronate: Six-year results from the Early Postmenopausal Interventional Cohort (EPIC) study. J Bone Miner Res 2002; 17: S134.

32. Schneider DL, Barrett-Connor EL, Morton DJ. Timing of postmenopausal estrogen for optimal bone mineral density. JAMA 1997; 277: 543-7.

33. Cauley JA, Zmuda JM, Ensrud KE, Bauer DC, Ettinger B. Timing of estrogen replacement therapy for optimal osteoporosis prevention. J Clin Endocrinol Metab 2001; 86: 5700-5.

34. Nelson HD, Rizzo J, Harris E, Cauley J, Ensrud K, Bauer DC, Orwoll E, for the Study of Osteoporotic Fractures Research Group. Osteoporosis and fractures in postmenopausal women using estrogen. Arch Intern Med 2002; 162: 2278-84.

35. Neele SJM, Evertz R, De Valk-De Roo G, Roos JC, Netelenbos JC. Effect of 1 year of discontinuation of raloxifene or estrogen therapy on bone mineral density after 5 years of treatment in healthy postmenopausal women. Bone 2002; 30: 599-603.

36. Talbot JR, Fischer MM, Farley SM, Libanati C, Farley J, Tabuenca A, et al. The increase in bone mineral density that occurs in response to fluoride therapy for osteoporosis not maintained after the therapy is discontinued. Osteoporos Int 1996; 6: 442-7.

37. Lindsay R, Scheele WH, Neer R, Pohl G, Adami S, Mautalen CA, et al. Sustained vertebral fracture risk reduction after withdrawal of teriparatide (recombinant human parathyroid hormone (1-34) in postmenopausal women with osteoporosis. Arch Intern Med 2004; 164: 2024-30.

38. Kaufman JM, Orwoll E, Goemaere S, San Martin J, Hossain A, Dalsky GP, et al. Teriparatide effects on vertebral fractures and bone mineral density in men with osteoporosis: treatment and discontinuation of therapy. Osteoporos Int 2005; 16: 510-6.

39. Bone HG, Hosking D, Devogelaer JP, Tucci JR, Emkey RD, Tonino $\mathrm{RP}$, et al. Ten years' experience with alendronate for osteoporosis in postmenopausal women. N Engl J Med 2004; 350: 1189-99.

40. Recker RR, Barger-Lux J. Risedronate for prevention and treatment of osteoporosis in postmenopausal women. Expert Opin Pharmacother 2005; 6: 465-77.

41. McCombs JS, Thiebaud P, MCLaughlin-Miley C, Shi J. Compliance with drug therapies for the treatment and prevention of osteoporosis. Maturitas 2004; 48: 271-87.

42. Caro JJ, Isaac KJ, Huybrechts KF, Raggio G, Naujoks C. The impact of compliance with osteoporosis therapy on fracture rates in actual practice. Osteoporos Int 2004; 15: 1003-8

43. Cosman F, Nieves J, Woelfert L, Formica C, Gordon S, Shen V. Parathyroid hormone added to established hormone therapy: effects on vertebral fracture and maintenance of bone mass after parathyroid hormone withdrawal. J Bone Miner Res 2001; 16: 925-31.

44. Pools HA, Felsenberg D, Hanley DA, Stepan J, Muñoz-Torres M, Wilkin TJ, et al. Multinational, placebo-controlled randomized trial of the effect of alendronate on bone density and fracture risk in postmenopausal women with low bone mass: results of the FOSIT Study. Osteoporos Int 1999; 9: 461-8.

45. Papaionnau A, Ioannidis G, Adachi JD, Sebaldt RJ, Ferko K, Puglia M, et al. Adherence to bisphosphonates and hormone replacement therapy in a tertiary care setting of patients in the CANDOO database. Osteoporos Int 2003; 14: 808-13.

46. Zafran N, Liss Z, Peled R, Sherf M, Reuveni H. Incidence and causes for failure of treatment of women with proven osteoporosis. Osteoporos Int 2005; 16: 1375-83.

47. Unson CG, Siccion E, Gaztambide J, Gaztambide S, Mahoney Trella P, Prestwood K. Nonadherence and osteoporosis treatment preferences of older women: a qualitative study. J Womens Health (Larchmt). 2003; 12: $1037-45$.

48. Rossini G, Bianchi G, Di Munno O, Giannini S, Minisola S, Sinigaglia $\mathrm{L}$, et al. Determinants of adherence to osteoporosis treatment in clinical practice. Osteoporos Int 2006; on line.

49. Cano A. Compliance to hormone replacement therapy in menopausal women controlled in a third level academic centre. Maturitas 1994; 20: 91-9.

50. Ravnikar VA. Compliance with hormone therapy. Am J Obstet Gynecol 1987; 156: 1332-4.

51. Castelo-Branco C, Figueras F, Sanjuan A, Vicente JJ, Martinez de Osaba MJ, Pons F, et al. Long-term compliance with estrogen replacement therapy in surgical postmenopausal women: benefits to bone and analysis of factors associated with discontinuation. Menopause 1999; 6: 307-11.

52. Ettinger B, Pressman A, Silver P. Effect of age on reasons for initiation and discontinuation of hormone replacement therapy. Menopause 1999; 6: 282-9.

53. Ryan PJ, Harrison R, Blake GM, Fogelman I. Compliance with hormone replacement therapy (HRT) after screening for postmenopausal osteoporosis. Br J Obstet Gynaecol 1992: 99: 325-8.

54. Doren M, Schneider HP. The impact of different HRT regimens on compliance. Int J Fertil Menopausal Stud 1996; 41: 29-39.

55. Sosa Henríquez M, Navarro Rodríguez MC, Segarra Sánchez MC, Cabrera Domínguez C, Limiñana Cañal JM, Hernández Hernández D, et al. Cumplimiento del tratamiento hormonal sustitutivo en la menopausia en una población canaria. Seguimiento durante 3 años. Rev Esp Enf Metab Oseas 1994; 3: 150-4.

56. Lombas C, Hakim C, Zanchetta JR. Compliance with alendronate treatmen in an osteoporosis clinic. J Bone Miner Res 2001; 15 (Supl. 1): S448. 
57. World Health Organization. Meeting Report. Adherences to long-term therapies. Policy for action. World Health Organization, 2001.

58. World Health Organization. Prevention and management of osteoporosis. World Health Organ Tech Rep Ser 2003; 921: 1-164.

59. Grupo de trabajo de la Sociedad Española de Investigaciones Óseas y Metabolismo Mineral (SEIOMM). Osteoporosis postmenopáusica. Guía de práctica clínica. Rev Clin Esp 2003; 203: 496-506.

60. Sosa Henríquez M, Filgueira Rubio J, López-Herce Cid JA, Díaz Curiel M, Lozano Tonkin C, del Castillo Rueda A, et al. ¿Qué opinan los internistas españoles de la osteoporosis? Rev Clin Esp 2005; 205 : 379-82.

61. Cortet B, Bera-Louville A, Gauthier P, Gauthier A, Marchandise X, Delcambre B. Comparative eficacy and safety study of etidronate and alendronate in postmenopausal osteoporosis: efect of adding hormone replacement therapy. Joint Bone Spine 2001; 68: 410-5.

62. Hernández Hernández JL, Riancho Moral JA, González Macías J. Bisfosfonatos intravenosos. Med Clin (Barc) 2005; 124: 348-54.

63. Cryer B, Bauer DC. Oral bisphosphonates and upper gastrointestinal tract problems: what is the evidence? Mayo Clin Proc 2002; 77: 1031-43.

64. Cohen SB. An update on bisphosphonates. Curr Rheumatol Rep 2004; 6: 59-65.

65. Reid IR. Bisphosphonates: new indications and methods of administration. Curr Opin Rheumatol 2003; 15: 458-63.

66. Sosa Henríquez M, Jódar Gimeno E. Bifosfonatos: principios básicos, farmacocinética y tolerancia gastrointestinal. En: Calaf i Alsina J, ed. Bifosfonatos y osteoporosis postmenopáusica. Barcelona: Scientific
Comunication Management; 2003. p. 113-21.

67. Schnitzer T, Bone HG, Crepaldi G, Adami S, McClung M, Kiel D, et al. Therapeutic equivalence of alendronate $70 \mathrm{mg}$ once-weekly and alendronate $10 \mathrm{mg}$ daily in the treatment of osteoporosis. Alendronate Once-Weekly Study Group. Aging (Milano) 2000; 12: 1-12.

68. Brown JP, Kendler DL, McClung MR, Adachi JD, Bolognese MA, et al. The efficacy and tolerability of risedronate once a week for the treatment of postmenopausal osteoporosis. Calcif Tissue Int 2002; 71: 103-11.

69. Rizzoli R. Long-term outcome of weekly bisphosphonates. Clin Orthop Relat Res 2006; 443: 61-5.

70. Bartl R, Goette S, Hadji P, Hammerschmidt T. Persistence and compliance with daily and weekly bisphosphonates in German women with osteoporosis. 5th European Congress on Clinical and Economic Aspects of Osteoporosis and Osteoarthritis (ECCEO). March 16-19, 2005. Roma (Italy). Póster nº 195.

71. Cramer JA, Amonkar MM, Hebborn A, Suppapanya N. Does dosing regimen impact persistence with bisphosphonate therapy among postmenopausal osteoporotic women? J Bone Miner Res 2004; 19 Supl1: S1.

72. Simon JA, Lewiecki EM, Smith ME Petruschke RA, Wang L, Palmisano JJ. Patient preference for once-weekly alendronate $70 \mathrm{mg}$ versus once-daily alendronate $10 \mathrm{mg}$ : a multicenter, randomised, open-label crossover study. Clin Ther 2002; 24: 1871-86.

73. Kendler D, Kung QWC, Fuleihan GE, Gonzalez Gonzalez JG, Gaines KA, Verbruggen N, et al. Patients with osteoporosis prefer once weekly to once daily dosing with alendronate. Maturitas 2004; 48: 243-51. 